\title{
PROBLÈMES \\ DE RÉGULARISATION ET D'AMÉNAGEMENT DU \\ DANUBE HONGROIS \\ ENTRE \\ SZOB ET MOHACS
}

PAR

D. IHRIG *

\section{Description caractéristique du secteur}

Le Danube hongrois entre Szob et Mohács représente environ un tiers du parcours du Danube dans le bassin des Karpates, dit Moyen Danube.

Sur la base de ses particularités morphologiques et hydrauliques, ce secteur peut se diviser en trois parties :

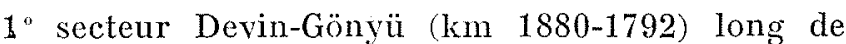
$88 \mathrm{~km}$, secteur de transition en voie d'exhaussement $(A)$;

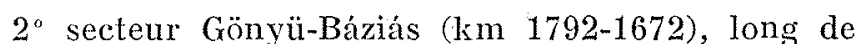
$720 \mathrm{~km}$, secteur à faible pente, rivière de plaine (B) ; puis,

$3^{\circ}$ le secteur Báziás-Turnu-Severin (km 1702-931), nouveau secteur de percée, long de $141 \mathrm{~km}$.

La division du secteur de plaine à faible pente Gönyü-Báziás (B) en deux parties par l'embouchure de l'Ipoly, signifie au fond en même temps la séparation de ces deux parties de plaine ((fig. 1). L'une d'elles, s'étendant de Gönyü jusqu'à l'embouchure de l'Ipoly ( $\mathrm{km} \mathrm{1708)}$ est la partie dite de la Petite Plaine $\left(B_{1}\right)$ tandis que l'autre, depuis le coude tournant le fleuve vers le sud ( $\mathrm{km}$ 1688) jusqu'à Báziás, est la partie dite de la Grande Plaine $\left(B_{2}\right)$. Entre ces deux parties s'intercale, près de l'embouchure de l'Ipoly, le secteur de la percée de Nagymaros, long de $27 \mathrm{~km}$. Ici, la percée de la région de montagne est entièrement achevée et la pente du Danube correspond parfaitement au caractère d'un fleuve de plaine.

Le secteur, long de $275 \mathrm{~km}$, Szob-Mohács du Danube ou plus exactement embouchure de l'Ipoly

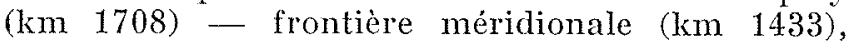
commence donc à l'embouchure de l'Ipoly avec un

* Ingénieur chef de section de l'Institut de Recherches des Ressources Hydrauliques, Budapest (Hongrie).

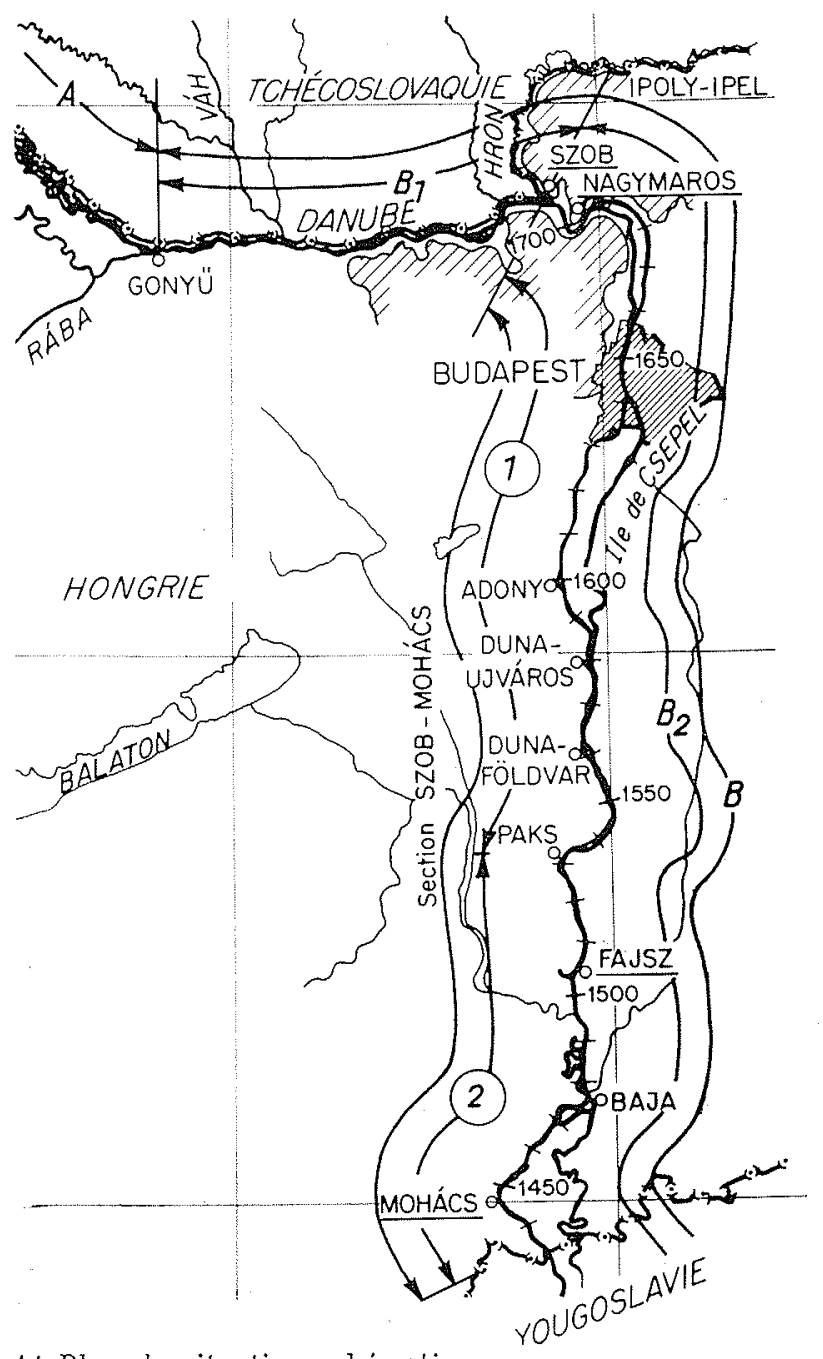

1/ Plan de situation schématique du Danube hongrois entre Szob et Mohács.

Schematischer Lageplan der ungarischen Donau zwischen Szob und Mohács. 


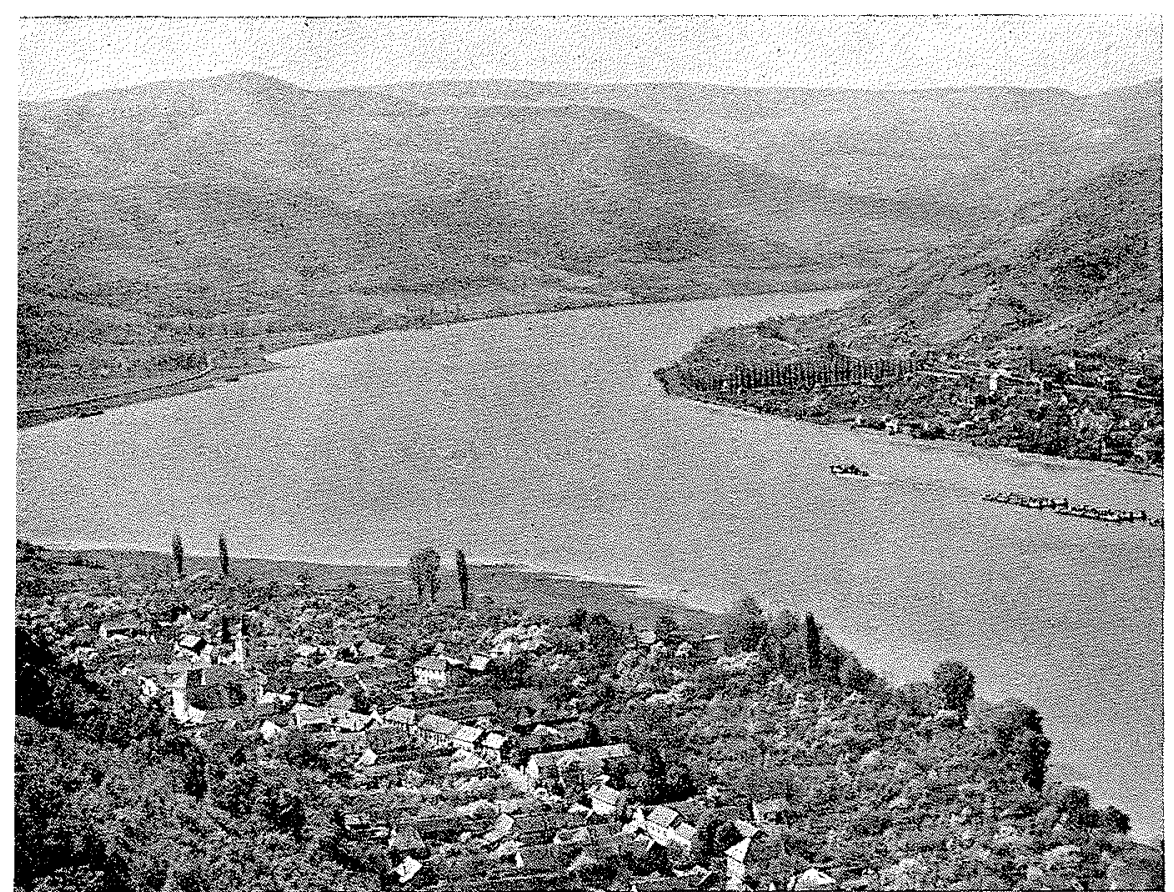

2/ Vue du secteur de percée de Nagymaros de la rive droite vers l'amont. Ansicht des Durchbruchabschnitts bei Nagymaros vom rechten Ufer stromaufwärts.

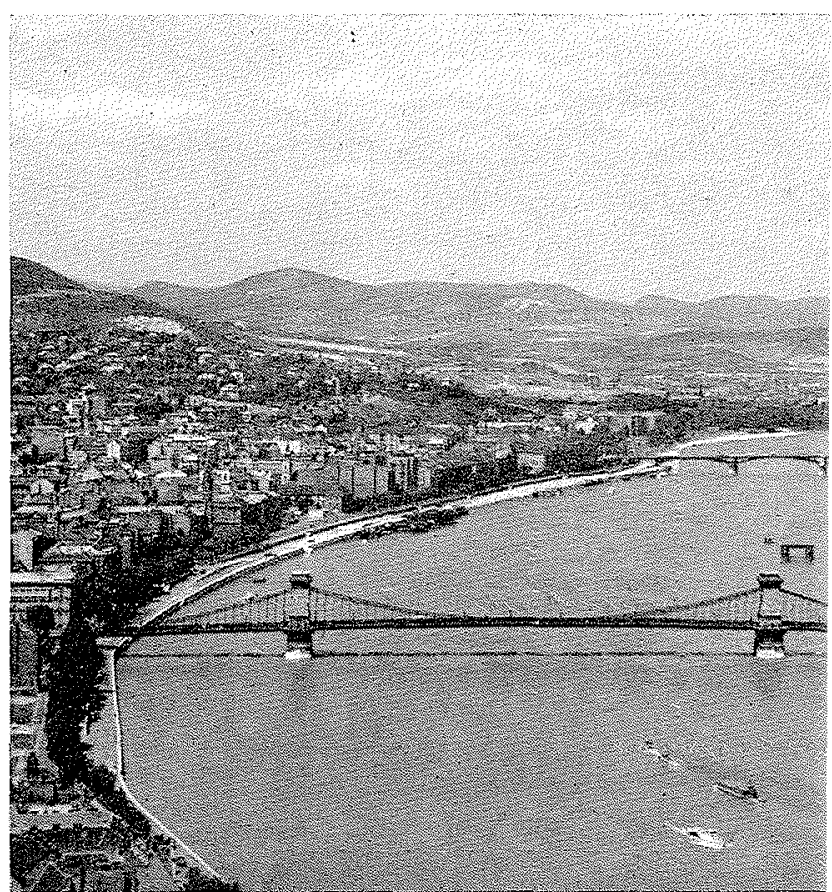

3/ Le Danube à Budapest.

Die Donau bei Budapest. secteur de percée déjà parfaitement développé de nos jours, ne troublant en rien le caractère de plaine du fleuve et quand même d'une beauté pittoresque (fig. 2). Le coude du Danube, ceint de montagnes, fournit en même temps l'emplacement de la première chute projetée sur le secteur purement hongrois du Danube, celle dite de Nagymaros.

A l'aval de la percée - en débouchant sur la Grande Plaine - le Danube a formé un cône de déjection. Celui-ci est complètement achevé de nos jours, mais ses traces se trouvent dans ces îles qui obligent le Danube, sur presque les deux tiers du secteur Szob-Mohács, à se diviser en branches. A peine entré sur la Grande Plaine, le Danube se divise en denx branches et l'île qu'il forme ainsi s'étend jusqu'à Budapest. Ayant transformé sa direction première, ouest-est, en intercalant le grand coude, en nord-sud, il conserve cette direction jusqu'à l'embouchure de la Drave. Aussi bien la direction dominante ouest-est que celle de nordsud sont imputables à des failles le long desquelles se sont affaissées la Petite Plaine à l'amont, resp. la Grande Plaine à l'aval de la percée de Nagymaros.

Sur le territoire de Budapest, le Danube forme d'abord encore des îles, puis, dans l'intérieur de la ville, il coule en ne conservant aucune trace des anciens îles et embranchements dans un lit enserré par les ouvrages de régularisation (fig. 3) jusqu'an tiers méridional de la ville. Ici, il se divise à nouveau en deux bras formant à leur tour une île longue de $50 \mathrm{~km}$ et large de $5 \mathrm{~km}$ en moyenne, l'île de Csepel. Des deux bras, celui de l'est est déjà canalisé de nos jours, une chute étant aménagée à chacune des extrémités avec écluse et usine hydro-électrique.

Aussitôt que la percée à l'amont de Budapest se trouve compensée et que le cône de déjection primitif a perdu sa tendance à l'exhaussement, la division caractéristique en bras cesse à une dis- lance de $80-90 \mathrm{~km}$ à l'aval de Budapest. Lc Danube fragmente son débit solide, mais puisqu'il transporte encore du débit roulé, la possibilité de la formation de bas-fonds et d'îles se maintient. Sur un certain trajet nous trouvons encore des îles.

Cependant, dans le tiers aval du secteur au-delà de Paks ( $\mathrm{km} \mathrm{1533),} \mathrm{l'aspect} \mathrm{du} \mathrm{fleuve} \mathrm{change.}$ Le Danube se met à y prendre les caractéristiques d'un parfait cours d'eau de plaine. Le débit roulé devient très fin et le fleuve transporte la majeure partie de son débit solide en suspension. Sa pente aussi diminue; elle n'est plus que de $5-6 \mathrm{~cm}$ par kilomètre, alors qu'elle était de $8-9 \mathrm{~cm}$ en moyenne sur les deux tiers amont du secteur. Sa force vive dépasse quand même la cohésion de la matière constituant les rives; il attaque donc celles-ci, les afouille, forme des méandres, transporte plus loin et dépose la matière lavée. Ce n'est plus la division en bras qui forme des îles, mais les méandres qui se rompent et les parties de lits découpées qui les embrassent (fig. 4).

Tandis que ce sont la végétation des montagnes longeant la rive droite et des couches de couverture minces déposées sur le gravier à l'aval de Budapest - respectivement les chaînes de loess arrivant sur la rive droite en plusieurs points jusqu'au Danube -.. qui donnent sur les deux tiers amont du secteur l'aspect caractéristique du Danube, ce sont, sur le tiers aval du secteur, les forêts luxuriantes des champs d'inondation sur. un sol riche en humidité des sédiments fins plus épais, des lits abandonnés et des îles s'étant formées par suite de coupures, qui évoquent l'aspect plein de fraîcheur de l'état primitif du Danube coulant en largeur (fig. 5 et 6). Ce n'est qu'en amont de Mohács, sur la rive droite d'un petit secteur, que les collines de loess atteignent le Danube, lui donnant avec des rives escarpées des concours prononcés. 

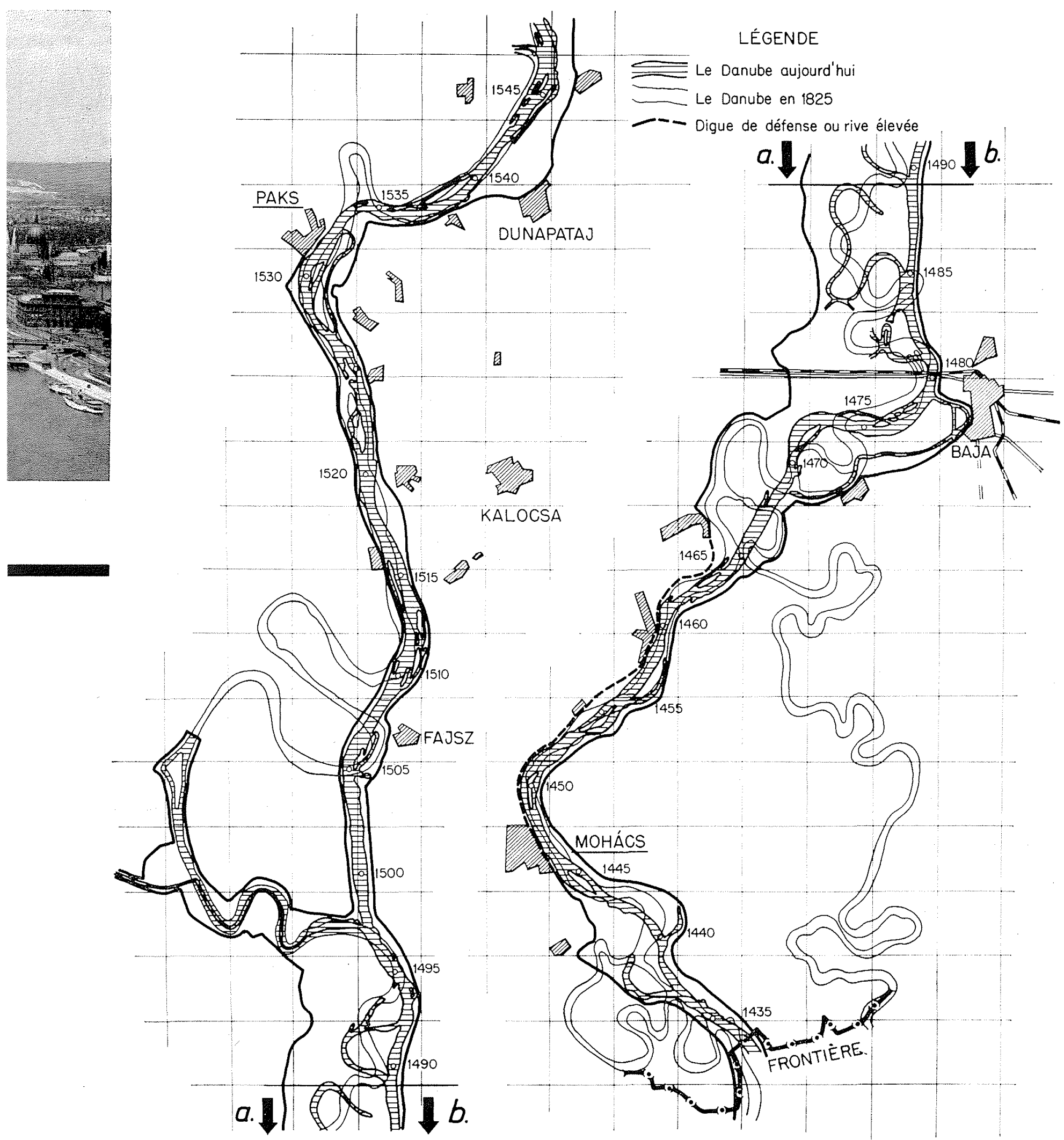

4/ Le secteur à lit mobile du Danube à Paks.

Comparaison de la situation avant la régularisation et celle stabilisée de nos jours.

Der Donauabschnitt mit beweglichem. Bett Paks.

Vergleich zwischen der Lage vor Ausführung der Regulierung

und der heutigen Lage nach Stabilisierung. 


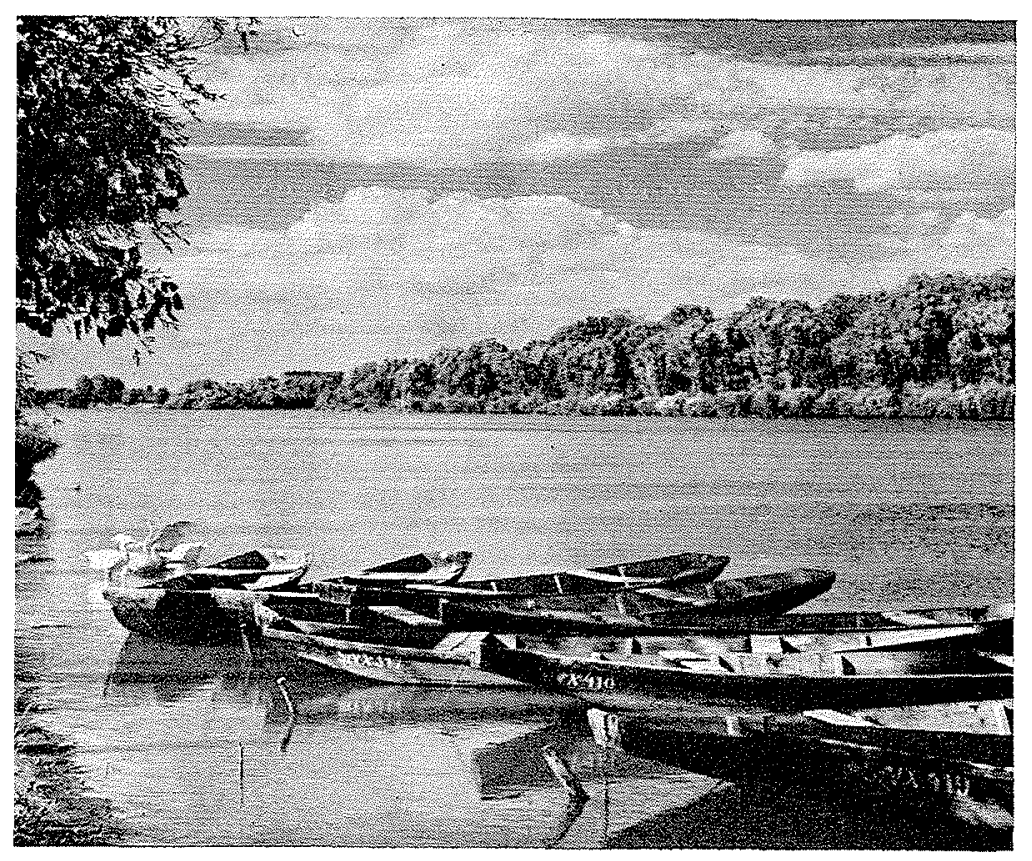

5/ Vue du secteur du Danube à lit stable entre Budapest et Paks (partie marquée 1 sur la fig. 1).
Ansicht des Donauabschnitts mit stabilem Bett zwischen Budapest und Paks Abb. 1, mit 1 bezeichneter Teil).

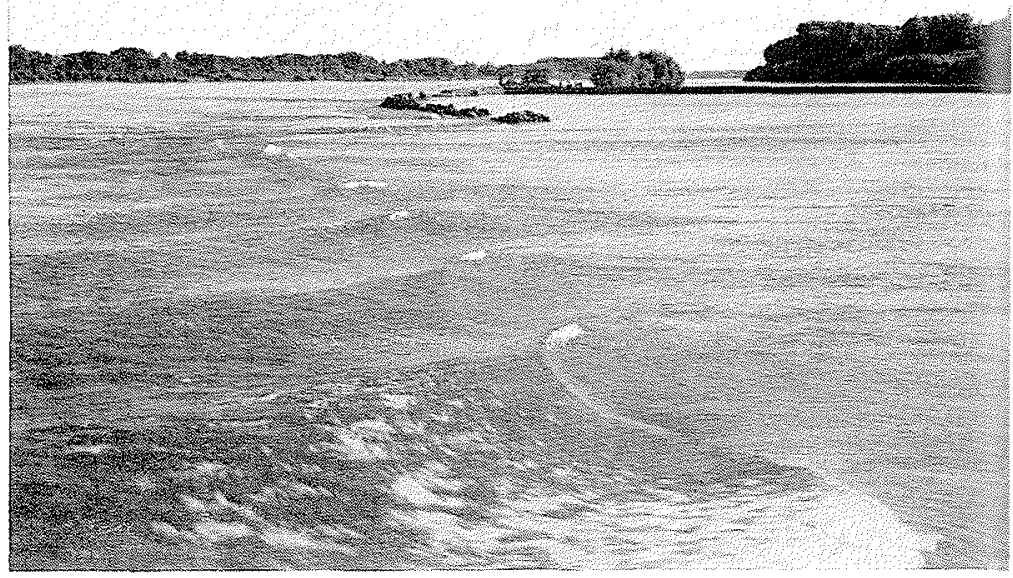

6/ Vue du secteur sinueux à lit mobile du Danube à l'aval de Paks. Au premier plan, ouvrage parallèle avec digue de jonction transversale en usage autrefois (partie marquée 2 sur la fig. 1).
Ansicht des gewundenen Donaum abschnits mit beweglichem Bett unterhalb Paks. Im Vordergrund ein ehemaliges Ixingswerk mit. Querverbindungsdeich (Abb. 1 , mit 2 bezeichneter Teil).

\section{Régularisation du secteur et les données qui l'influencent}

Dans leur état naturel, les rivières sont généralement des éléments nocifs ou peu utiles de la nature. Il en est ainsi surtout pour leurs secteurs de plaine, à pente faible. I.eurs courants endommagent leurs vallées et assurent à peine la navigation dans leurs lits dégénérés ou en train de le devenir. Le but de la régularisation est donc d'assurer et de maintenir les conditions d'écoulement réduisant au minimum les dommages et assurant le mieux l'utilisation de la rivière.

On distingue d'habitude trois débits caractéristiques des rivières -- eu égard aussi à leurs effets précités : les hautes caux débordant du lit origine] creusé dans le terrain naturel, les eaux moyennes s'écoulant dans ce lit originel dont la formation est à leur attribuer principalement; enfin, les basses eaux. L'écoulement de ces débits est assuré respectivement par les lits majeurs des eaux moyennes et basses, limitées naturellement ou artificiellement, et leur aménagement technique permettant de supprimer les dégâts et d'assurer la navigation consiste en régularisations des hautes eaux, des moyennes et basses eaux.

Sur le secteur de plaine du Danube qui nous occupe, la pente du fleuve est de 6 à $9 \mathrm{~cm} / \mathrm{km}$, son débit de 500 à 10000 , en moyenne de $2120 \mathrm{~m}^{3} / \mathrm{s}$. Sur un pareil secteur de plaine, les trois méthodes de régularisation ont toutes une grande importance.

Nous parlerons d'abord de la régularisation en eaux moyennes ayant pour objet leur lit compris entre les rives originelles. Son but est d'assurer l'équilibre en évacuant l'eau, la glace et le débit solide transportés par la rivière, en d'autres termes, d'assurer qu'il ne se produise ni emmagasinement de l'eau, ou son rabattement par aspiration, ni bouchon ou embâcle de glace, ni exhaussement ou approfondissement du lit. Si l'on réussit à assurer l'équilibre par la régularisation en eaux moyemes, la largeur et la profondeur requises pour la navigation seront généralement assurées. Cette régularisation en eaux moyennes ne doit être complétée qu'au cas où elle ne parviendrait pas à assurer, à l'époque des basses eaux, la profondeur d'eau nécessaire à la navigation.

C'est ainsi que la régularisation en basses eaux se fait, comme complément de celle en eaux moyennes, dans l'intérêt de la navigation.

Le mode de régularisation du lit entre les rives originelles est déterminé, en dehors des caractéristiques hydrauliques de la rivière, en grande partie par la nature des matériaux du lit et par celle du débit solide.

Nous avons déjà noté qu'après avoir percé à Nagymaros, le Danube a charrié une partie de la matière déposée à l'amont par la Petite Plaine et l'a déposée en débouchant sur la Grande Plaine. Ici, il a construit un important cône de déjection et les nombreuses îles existant encore de nos jours jusqu'à Paks sont les restes de l'ancien écoulement en bras du Danube. Celui-ci coule sur le cône de déjection et e'est pour cela que son lit est du gravier difficile à désagréger; le lit est stable. Mais, à l'aval de Paks, le fleuve coule dans un lit formé dans des sédiments fins qui se désagrègent donc facilement; le lit est sinueux.

Le débit roulé du Danube, de nos jours, comprend à Devin des grains allant jusqu'à la taille d'une tête d'enfant. Ce débit solide est influencé jusqu'à l'embouchure de l'Ipoly, de diverses façons, par les affluents du fleuve; jusqu'à Budapest, les plus gros grains vont encore jusqu'à la taille d'une noix; plus loin, ils diminuent sans cesse; aux environs de Paks, le débit solide n'est plus que du 
le diamètre atteigne encore le millimètre. Sur le sable rude, et il s'amenuise tellement jusqu'à Mohács que l'on y trouve à peine des grains dont secteur à l'aval de Paks, c'est en suspension que le Danube transporte la majeure partie de son débit solide.

Compte tenu de la diversité de la matière du lit et du débit solide, le secteur Szob-Mohács peut se diviser - comme il a été déjà dit dans la description sommaire du secteur — en deux parties (1 et 2 sur fig. 1):

$1^{\circ}$ la partie à l'amont de Paks, à lit stable transportant du débit roulé; et

$2^{\circ}$ la partie sinueuse à l'aval de Paks, à lit mobile transportant du débit en suspension, la partie de plaine du secteur.

Aux limites des secteurs, non seulement les conditions du débit solide, mais aussi le caractère morphologique du fleuve change.

Sur la partie amont du secteur marqué 1 , à lit stable (km 1708-1533), le lit est constitué de gravier rude; en même temps, le débit solide transporté est déjà à gravier plus fin. Le fond de gravier est immobile; l'eau ne peut pas désagréger le fond résistant; elle use plutôt les rives, élargit le lit. C'est pour cela que le Danube se caractérise ici par un long parcours en ligne droite, par un lit large se divisant en bras.

Sur la partie aval marquée 2 ( $\mathrm{km}$ 1533-1433), la matière du lit est déjà aussi fine que le débit solide transporté. Partant, le Danube est sinueux, se caractérise par des méandres; le lit est bien encaissé et tend à s'approfondir.

Il résulte de ce qui précède que le lit originel doit être traité sur chacune des parties du secteur suivant d'autres principes.

La régularisation aux hautes eaux était dans le temps identique à la solution de défense contre les crues qui protégeait par des levées de protection contre les inondations, construites sur chacune des rives, les terrains pouvant être cultivés et les agglomérations de la vallée du fleuve. La superficie ainsi protégée par des levées de protection de $450 \mathrm{~km}$ est de 430000 ha sur le secteur SzobMohács. La défense contre les crues incombait dans le passé à ces milieux restreints dont les terres furent sauvées de l'inondation. Ceux-ci ne voyaient que la protection contre l'inondation et ne prêtaient pas attention au rôle des levées dans la formation du lit, dans l'évacuation des crues et dans la régularisation en hautes eaux. Mais souvent ils ne pouvaient d'ailleurs pas le faire, du fait qu'à cette époque le lit des eaux moyennes n'était pas encore régularisé (voir les lignes des levées de protection contre les crues, sur fig. 4).

Sur la partie à l'amont de Budapest, des levées de défense contre les crues n'étaient nécessaires qu'en quelques endroits, les rives étant hautes; mais à l'aval, le lit majeur est partout - sauf où les collines de loess arrivent jusqu'au Danube limité par des levées de défense contre les crues. Au point de vue de l'évacuation de l'eau et de la glace, celles-ci laissent cependant beaucoup à désirer.

\section{Régularisation du lit aux moyennes et basses eaux}

Sur le secteur en question du Danube, le but principal de la régularisation en eaux moyennes était d'assurer l'évacuation sans entraves de la glace. Ce n'est que plus tard, lorsque la navigation se développa, que l'amélioration des conditions de celle-ci se situa au premier plan. Dans l'état naturel du fleuve - comme on le voit sur la figure 4 aussi - la glace s'est arrêtée entre les îles, dans les courbes, dans les rétrécissements, entre les basfonds des rives et du lit; des embâcles se produisirent, occasionnant des inondations dévastatrices. Une de celles-ci fut la crue glacée de 1838 qui a détruit en grande partie Budapest, laquelle se composait alors des villes de Buda et de Pest.

Nous plaçons le début des travaux de régularisation $d u$ lit du troncon Szob-Mohács à cette époque, bien que l'on ait commencé dès 1820 à couper les mŕandres à l'aval de Paks. On a effectué tout d'abord la régularisation du secteur du Danube à Budapest el environs en fermant la branche gauche de l'ile de Csepel formée par le Danube et en régularisant la branche droite en vue de la mieux approprier pour l'évacuation des glaces, Après la crue de 1876, également glacée, c'est depuis Budapest jusqu'à Adony (km 1598), puis, après les crues de la fin du siècle, jusqu’à Fajsz (km 1508) que l'on a régularisé le lit du Danube en mème temps que sur quelques autres secteurs isolés plus petits du fleuve. Le premier projet d'ensemble pour le Moyen Danube tout entier et par suite pour le secteur Szob-Mohács, qui cn fait partie, a été dressé également en 1893.

Dans le passé, le plan de régularisation n'a guère été exécuté uniformément et de façon continue, mais suivant les besoins, et par suite des guerres de notre sicele, les travaux furent interrompus pendant des périodes assez longues. C'est ainsi que, dans son ensemble, le secteur ne peut pas satisfaire aux besoins de la navigation ni assurer sans dégâts l'évacuation des glaces. Dans la décennie écoulée, on a dressé pour le secteur entier un nouveau plan d'ensemble complet répondant cette fois-ci aux conditions et exigences changées. La réalisation du projet comme complément de la régularisation exécutée dans le passé, est en cours et sera poursuivie aussi dans l'avenir.

Ces travaux de régularisation sont censés satisfaire aux besoins accrus de la navigation et reposent sur une base triple : détermination appropriée du niveau de l'eau, de la largeur et de la profondeur de la régularisation.

Un niveau d'eau de régularisation uniforme est fixé sur le secteur depuis 1930. Depuis 1958, ce sont les niveaux d'eau calculés suivant les propositions de la Commission du Danube, dont on se sert pour déterminer le niveau des basses eaux pour la navigation, resp. le niveau de régularisation. Ces niveaux correspondent aux niveaux des sept principales échelles du secteur dont la durée de fréquence est de $94 \%$ en moyenne. Le profil en long détaillé du niveau de régularisation entre ces échelles est établi en se servant des niveaux d'eau effectivement fixés.

La largeur de la régularisation a été fixée par le projet de 1893 à $450 \mathrm{~m}$, mais fut réduite, sur la base des expériences, à $400 \mathrm{~m}$ dans les projets ultérieurs. Sur ce secteur, le principe fondamental de la régularisation est surtout l'application de profils transversaux variables, la largeur étant de $400 \mathrm{~m}$ dans les nouvelles inflexions et de $350 \mathrm{~m}$ au point culminant de la courbe.

La profondeur de la régularisation fut fixée à 


\section{IHRIG}

$3 \mathrm{~m}$ en dessous du niveau de régularisation pour la partie à l'amont de Budapest et à $3,50 \mathrm{~m}$ à l'aval, mais, sur le secteur à cataractes du Bas Danube, cette profondeur n'est pas encore disponible pour la navigation maritime. Cependant, cette profondeur satisferait non seulement aux besoins de la navigation, mais elle donnerait aussi une sécurité suffisante du point de vue de l'évacuation des slaces.

Sur le secteur à lit stable embouchure de l'IpolyPaks (1 sur la fig. 1), on doit distinguer trois parties :

a) Dans la percée de Nagymaros, la profondeur de $3 \mathrm{~m}$ par eaux basses est partout assurée pour la navigation;

b) Depuis le secteur de la percée jusqu'à Budapest, le fleuve a développé son profil transversal en largeur; l'eau en se répandant a déposé son đébit solide, des bas-fonds se sont produits et la profondeur nécessaire à la navigation n'était guère assurée. Par ailleurs, cette partie était susceptible, encore au début du siècle, de produire des embâcles. Le premier projet de régularisation de 1893 a prescrit la fermeture des branches secondaires et le resserrement, à l'aide d'ouvrages parallèles, des secteurs larges du lit. Ce n'est qu'après la seconde guerre mondiale, en 1949, que fut dressé un plan en vue d'une amélioration radicale suivant la méthode des profils transversaux variables. Puisque sur cette partie le lit n'a pas encore été modifié par la régularisation en eaux moyennes, la méthode de Girardon consiste ici, non pas en une régularisation en basses eaux, mais en une régularisation s'étendant au lit tout entier. On a déterminé la ligne de courant la plus avantageuse; en fermant les lits secondaires, on réalisait le lit uniforme; sur la rive concave, on dirigeait la ligne du courant au moyen d'ouvrages parallèles et on resserrait le lit sur la rive concave avec des épis.

c) De Budapest à Paks, ce n'est pas tant à cause des obstacles à la navigation, mais pour éliminer les dégâts causés par les crues, que la régularisation est nécessaire. Nous parlerons plus loin de la régularisation du lit majeur; quant à celles en eaux moyennes et basses, elles sont régies par les mêmes considérations que celles énoncées pour les parties précédentes. La figure 7 présente le projet de régularisation d'une partie du secteur prise au hasard, resp. l'exécution partielle des travaux. Ceux-ci s'appuient sur la régularisation déjà exécutée et la complètent. Cette dernière est basée également sur le principe des profils transversaux variables.

Le secteur sinueux Paks-frontière à lit mobile (2 sur la fig. 1) avait et a encore de nos jours une forte tendance à produire des crues occasiomnées par les embâcles. Dans les courbes, le brusque changement de direction forçait la glace à s'arrêter, tandis qu'après leur coupure, c'est dans celles-ci que le lit a dégénéré; des bas-fonds se produisaient. L'écoulement de l'eau étant lent, la glace s'arrête facilement. Dix-huit des grandes courbes furent coupées encore au siècle dernier; mais les coupures ne se sont pas développées de facon à supprimer le danger d'embâcles.

Le projet de régularisation de 1893 déjà cité a prévu pour ce secteur également la régularisation aux profils constants. Puisque, suivant l'expérience, la glace ne s'arrète pas et que des embâcles ne se produisent guère là où la profondeur en dessous du zéro est constamment $3 \mathrm{~m}$, on a fixé la largeur de la régularisation de manière à assurer que, dans le chenal de navigation, la profondeur soit au moins de $3 \mathrm{~m}$. En vue d'améliorer les conditions d'écoulement de l'eau et de la glace, de stabiliser le lit et les rives, d'assurer le chenal de navigation, ce projet prévoit des ouvrages parallèles pour rétrécir le lit, pour consolider les rives des défenses de berges, pour prévenir le partage des eaux lors des fermetures de bras secondaires, pour assurer la profondeur voulue des dragages, pour supprime: les courbes aiguës des coupures, ponr raccorder les ouvrages parallèles de digues transversales et, seulement dans des cas exceptionnels, des épis.

Au cours des travaux actuellement en cours et à poursuivre dans l'avenir, on construit dans la ligne de régularisation, autant que possible dans la continuation des rives en défense des berges bien tracées, des ouvrages de guidage, et on remblaie en faisant déposer la vase dans les parties de lit coupées par les digues transversales ou bras secondaires.

Des ouvrages complémentaires de régularisation en basses eaux ne devaient être construlits qu'au droit des coupures entre les $\mathrm{km}$ 1505-1480.

\section{La régularisation en hautes eaux}

Les plus hauts niveaux de crues glacées des derniers cent ans sont comparés, sur la figure 8 , aux plus hauts niveaux des crues exemptes de glaces de la même époque. La crue estivale de 1954 a dépassé aux échelles du Danube moyen, à l'exception de quelques-uns, les niveaux des crues exemptes de glaces survenues jusqu'ici; c'est donc au niveau de celles-ci que nous rapporterons les plus hauts niveaux des crues glacées. La figure montre, rapportés à ce niveau d'eau (à l'axe horizontal marqué 1) séparément les plus hauts niveaux de la crue glacée de mars 1956 (lignes marquées 2 et 3 ) et ceux des crues glacées antérieures (ligne marquée 4), l'année de la crue étant inscrite pour. chacun des maximums de ces derniers. La figure montre clairement que les crues glacées sont dangereuses - à la différence du secteur voisin d'amont - sur tout le secteur Szob-Mohács; mais les plus dangereuses sont sur le secteur à l'aval de Budapest jusqu'à la frontière. Il y a des endroits (Dunaföldvár, $\mathrm{km} \mathrm{1660)} \mathrm{où} \mathrm{la} \mathrm{crue} \mathrm{glacée} \mathrm{a} \mathrm{dépassé}$ de $2,5 \mathrm{~m}$ et, s'il n'y avait pas eu de ruptures de digues en 1956 , de $3,4 \mathrm{~m}$ (au $\mathrm{km}$ 1507) le maximum des crues exemptes de glace.

Sur les figures 9 et 10 , nous présentons quelques photos de la glace du Danube à l'aval de Budapest.

Les causes de ces crues glacées doivent être cherchées beaucoup plus loin que dans des défauts éventuels du lit des eaux moyennes. Il y a des causes fondamentales qui proviennent des données géographiques et météorologiques de ce secteur.

W. Lászlóffy a démontré dans son étude des crues glacées que sur ce secteur les embâcles et les crues glacées s'expliquent par des causes géographiques constantes, tandis que leurs dimensions catastrophiques sont à attribuer au régime défavorable des facteurs météorologiques.

Un facteur géographique constant est le fait que, depuis l'embouchure de l'Ipoly jusqu'à la Drave, le Danube hongrois n'a pas d'affluent dont les hautes eaux de la fonte des neiges détruiraient, iompraient la glace, comme c'est généralement lè 


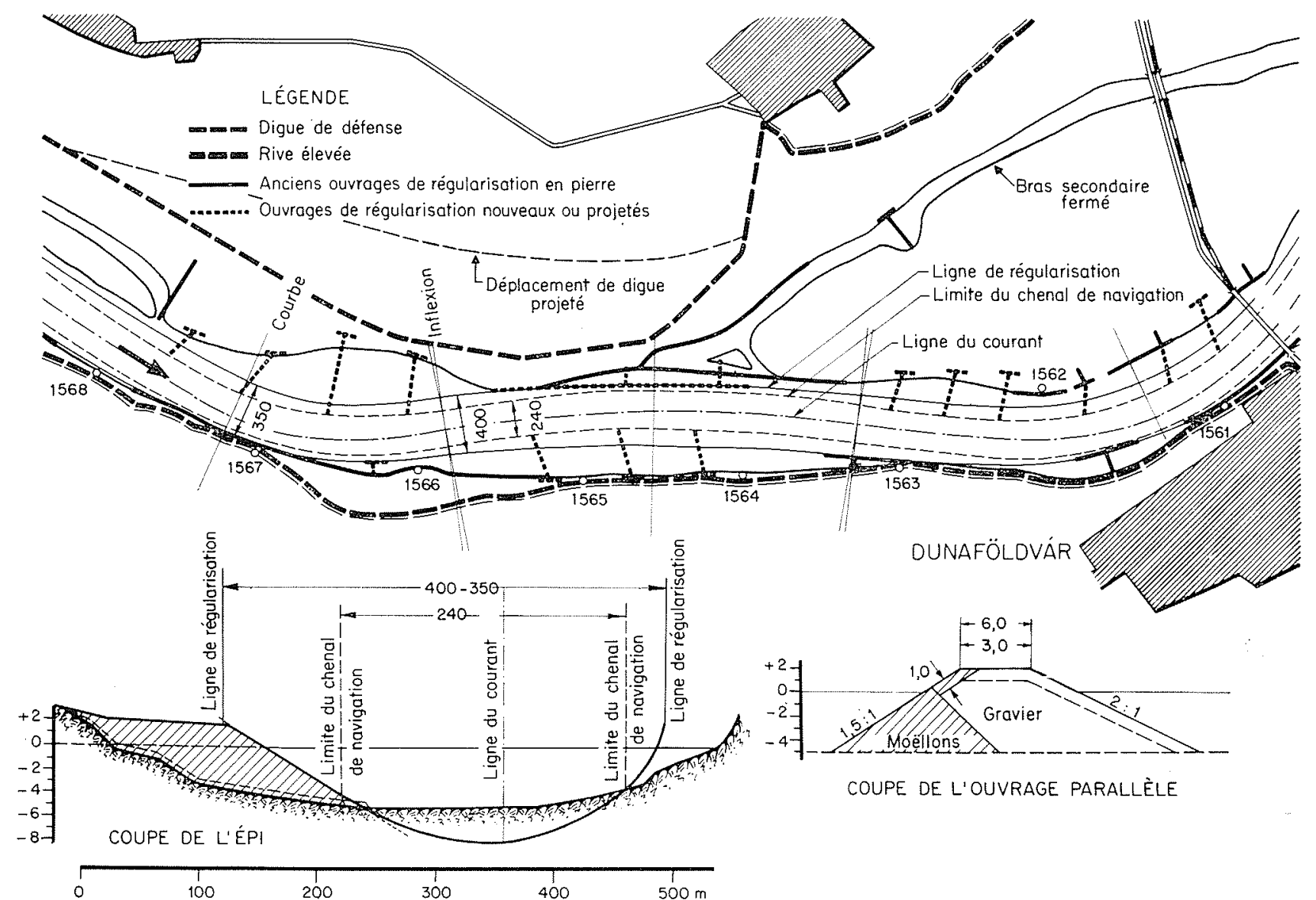

7/ Disposition de principe de la régularisation du secteur à lit stable en vue de faciliter aussi l'écoulenient des crues glacées. Prinzip der Regulierung des Abschnitts mit stabilem Bett, die auch einen besseren Abfluss des eisführenden Hochwassers ermöglichen soll.

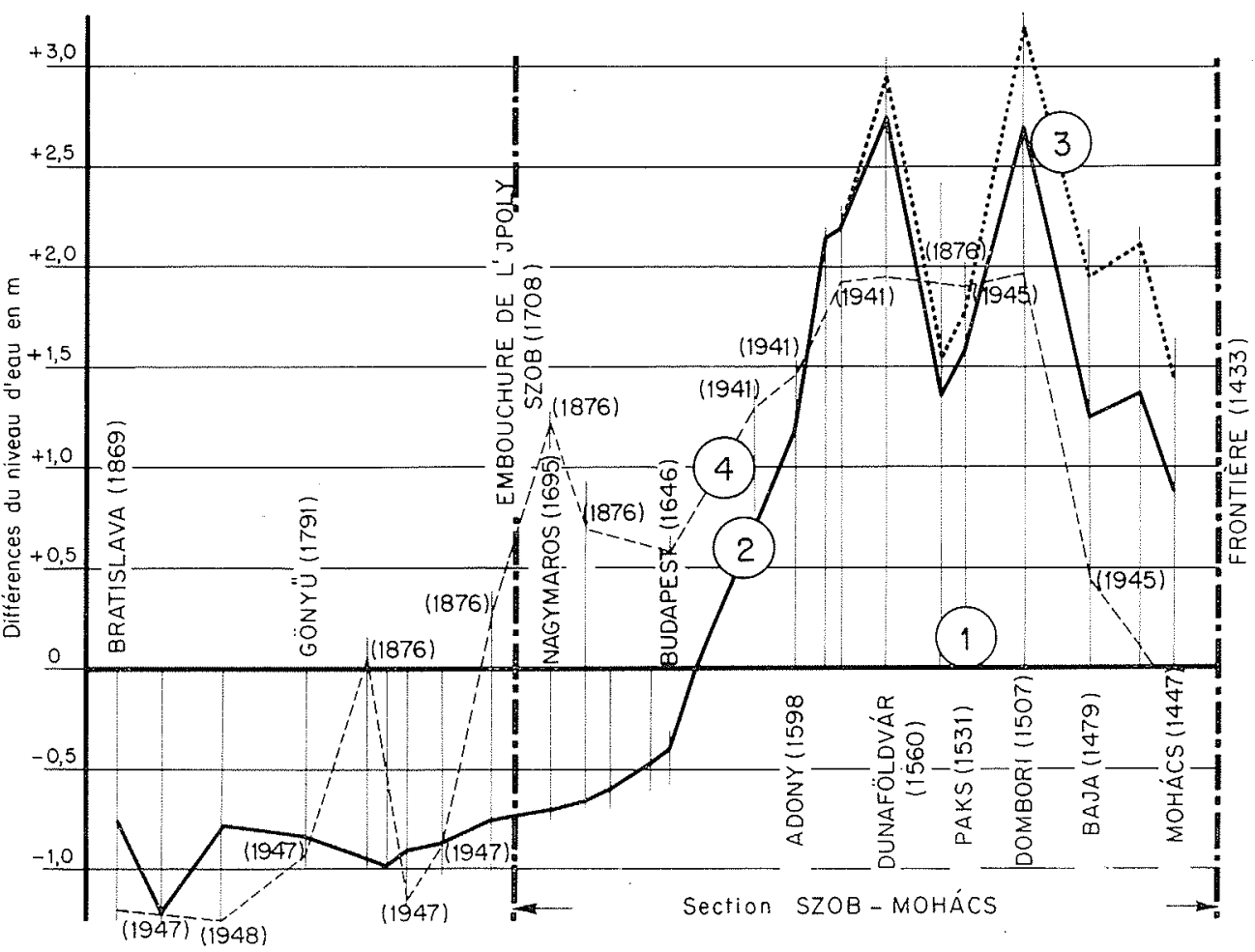

8/ Niveaux des crues glacées de 1956 et d'auparavant au-dessus des plus hauts niveaux exempts de glace (de 1954). L'axe horizontal (1) représente le niveau de crue le plus élevé (de 1954). (2) Niveanx effectifs les plus élevés de la crue glacée de 1956 . (3) Niveaux les plus élevés de la crue de $1956 \mathrm{cal}-$ culés en supposant qu'il n'y aurait pas eu de ruptures de digues. (4) Niveaux des crues glacées les plus élevées d’avant 1956 au-dessus de [1].

Spiegelhöhen der eisführenden Hochwasser von 1956 und früher über den höchsten eisfreien Wasserständen (Don 1954). Die horizontale Achse (1) stellt den höchsten Hochwasserstand dar (1954), (2) die wirksamen höchsten Wasserstände des eisführenden Hochwassers von 1956. (3) stellt die höchsten Wasserstände des Hochwassers von 1956 dar, bei deren Berechnung von der Annahme alusgegangen wurde. dass kein Deichbruch stattfand. (4) entspricht den über (1) liegenden höchsten Wasserständen der eis/ührenden Hochwasser von 1956 . 
Vue de l'extrémité aval du secteur du Danube représenté sur la figure 7 , avec le pont de Dunaföldvár lor's de l'embâcle de 1956.

Das stromabwärtige Ende des auf $A b b .7$ dargestellten Donauabschnitts mil der Brïcke von Dunaföldvar während des Eisstaus von 1959.
10) Montagne de glace pressée sur la rive au $\mathrm{km} 1493$ du Danube, 3 jours après le commencement de la débàcle, lorsque l'eau vient de revenir du champ d'inondation dans le lit.

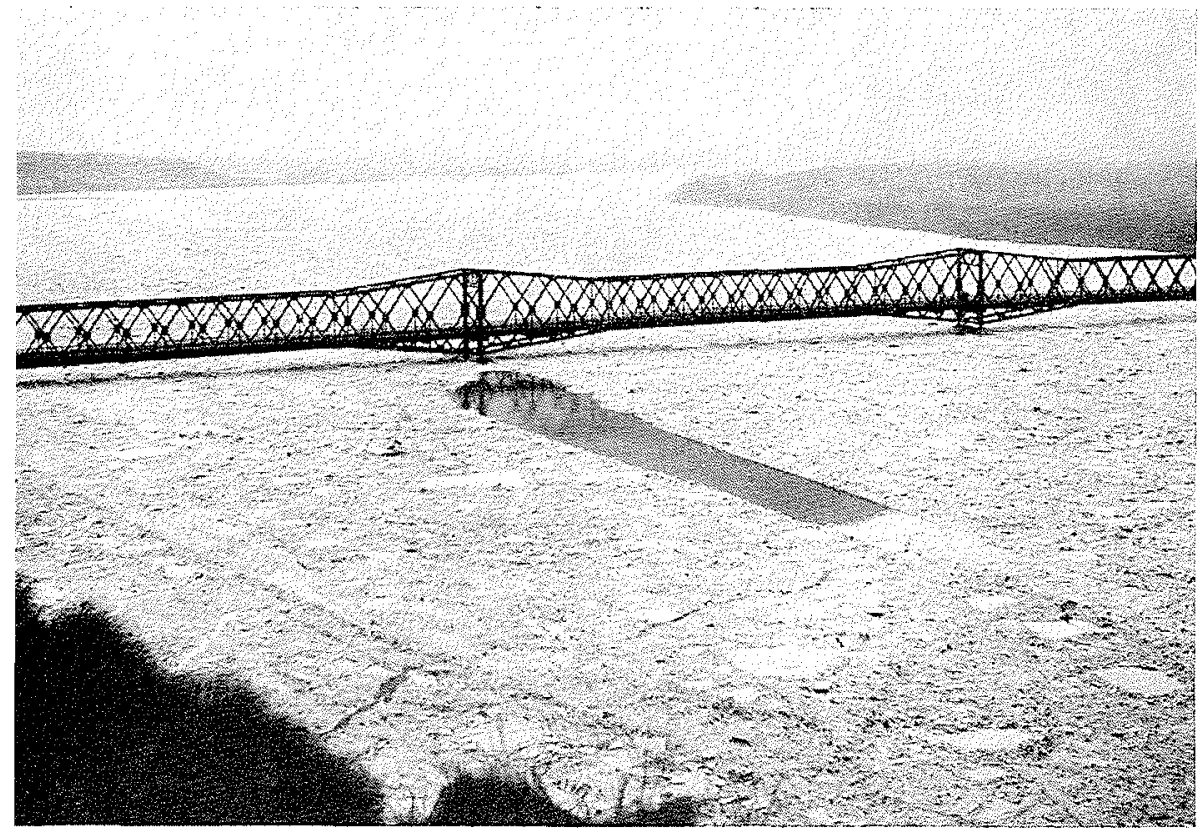

Ein Eisberg, der 3 Tage nach Beginn des Eisgangs, kurz nach dem Zurüclifyessen des Wassers aus dem tobershuemmangsgebiet in das Bett, bei km 1493 gegen das Ufer der Donau gedrückt wird.

cas sur le Haut Danube, voire sur le secteur de la Petite Plaine du Moyen Danube ou à l'aval de la Drave.

Le temps qu'il fait est ce facteur variable naturel qui peut rendre le régime des glaces catastrophique. Si c'est à l'ouest sur le cours supérieur du Danube que la hausse de la température commence et qu'au moment du départ de l'onde de crue se produisant sur le Haut Danube, la température ait été encore au voisinage du zéro sur la plaine hongroise ou qu'elle y soit tombée après un début d'adoucissement - le Danube étant toujours bloqué - de nouveau en dessous du point de gelée, l'onde de crue partie du Haut Danube y trouve des masses de glace soudées ensemble et aux rives. Les embâcles de glace fripées se mettant en mouvement sous l'effet de l'onde de crue peuvent provoquer des remous, et même des remous catastrophiques s'ils barrent le chemin à l'eau en des endroits où elle ne peut pas contourner par les champs d'inondation, par le lit majeur, l'embâcle du lit des eaux moyennes et ne trouve pas d'issue (par exemple, si le champ d'inondation est très étroit ou qu'il s'y trouve des obstacles: arbres touffus, digues transversales, levées de routes, etc.).

L'examen des crues glacées catastrophiques montre qu'au cours des derniers cent ans les ruptures de digues qu'elles provoquèrent se concentrent autour de certains nœuds, là où le lit majeur est étroit, sa largeur réduite. Si un embâcle se produit par suite d'une cause quelconque dans un tel secteur étroit ou à son aval, la situation catastrophique est due à l'étroit champ d'inondation. De pareils secteurs, nous en trouvons - du fait de I'irrégularité déjà mentionnée du lit majeur enserré entre les levées de protection contre les crues -sur le secteur du Danube à l'aval de Budapest, surtout entre des kilomètres 1670-1550, 1530-1490 et $1470-1450$.

Avec une régularisation adéquate en eaux moyennes, on peut faciliter l'évacuation des glaces mème dans les conditions naturelles les plus défavorables, comme nous l'avons déjà signalé en traitant de celles-là. Et avec une régularisation adéquate du lit majeur, avec un tracé approprié des levées et en éliminant les obstacles à l'écoulement dans les champs d'inondation, on peut procurer une issue à l'eau en remous du fait de l'embâcle.

En effet, si un embâcle se produit quelque part dans la glace bloquée, les dimensions du lit majeur ont une importance extraordinaire. Celles-ci sont adéquates si, en cas d'embâcle, l'eau peut s'écouler en dehors du lit des eaux moyennes, sur le champ d'inondation, à un niveau laissant une marge de sécurité suffisante sous la crête des levées de défense contre les crues.

En dehors du rôle d'empêcher les inondations, une tâche importante incombant aux levées de protection contre les crues est d'aider l'évacuation des glaces et des hautes eaux. Et ceci n'est possible que si le lit majeur a une capacité d'évacuation suffisante, même si la glace bloque le lit des eaux moyennes. Généralement, il est à souhaiter que, de chaque côté du lit des eaux moyennes, le champ d'inondation ait la même largeur que celui-ci et que la ligne de courant des hautes eaux ne diffère pas considérablement de celle des eaux moyennes. A titre d'exemple, la figure 7 montre, en même temps que la régularisation des eaux moyennes et basses, la démolition et le déplacement de la levée de protection contre les crues sur un des secteurs provoquant des hauteurs d'eau catastrophiques, ceci en vue de la régularisation du lit majeur. Il s'y ajoute la libération d'obstacles à l'écoulement des champs d'inondation bordant le lit des eaux moyennes. Ceci peut s'obtenir en abattant des arbres et en aménageant dans les levées de routes plus hautes que le niveau de crue, des aqueducs d'une capacité suffisante.

Mais dans les travaux de régularisation au sec. 
teur à l'aval de Budapest, on prend en considération non seulement leur effet sur l'évacuation de la glace et de l'eau en remous, mais aussi sur l'écoulement des crues et sur la formation du lit, tant en les exécutant qu'en les projetant, en effectuant des essais sur modèles réduits en vue de trouver la forme à donner au lit majeur. L'élargissement brusque du lit majeur fait diminuer la profondeur du lit des eaux movennes, mais celui-ci se forme encore irrégulièrement au cas où les lignes de courant des hautes eaux et moyennes eaux diffèrent les unes des autres.

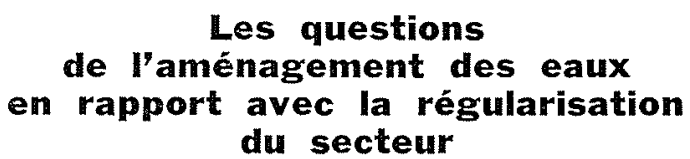

Il faut consacrer quelques mots aux questions de l'aménagement, de l'utilisation des eaux du Danube sur ce secteur. Il faut examiner les questions de la navigation, de la mise en valeur de l'énergie hydraulique et de l'approvisionnement en eau.

Dans le passé, c'est la navigation qui était le principal utilisateur du Danube. En effet, sur un cours d'ean naturel, les plus importants obstacles pour la navigation sont la glace et les bas-fonds.

Les conditions de slace du secteur se caractérisent par les durées moyennes et extrêmes de la présence de la slace, du fleuve bloqué par la glace et de celle de charriage de glacons. Les chiffres étaient pour les trois caractéristiques moyennes et extrêmes 44, 40, 16 resp. 100, 83,56 jours dans la période de 1899-1900 à 1953-1954. En comparant ces données avec celles des autres secteurs du Danube, nous pouvons dire qu'après le secteur Braila-Sulina, il se montre, avec celui allant jusqu’à la Drave, le plus désavantageux. Les travaux de régularisation, dont le but principal était, comme nous l'avons vu, d'améliorer l'évacuation des glaces, tant du point de vue de la navigation que de celui de la défense contre les crues, ont beaucoup fait pour atteindre ces buts, mais ne pouvaient pas encore résoudre l'écoulement sans entraves des glaces. En fin de compte, la durée movenne de la saison de navigation est de 321 jours sur ce secteur, ce qui est satisfaisant si on la compare à la moyenne de 322 jours du parcours tout entier à l'aval de Szob.

Cependant, il y a encore sur le secteur des basfonds empèchant de mettre completement à profit cette saison de navigation. Si nous considérons la navigation avec remorques de $1200 \mathrm{t}$, nous rencontrons sur les secteurs Szob-Budapest et Dunaföldvár-frontière, lors des plus bas niveaux de navigation, des manques de profondeur par rapport aux $25 \mathrm{dm}$ nécessaires, alors que le secteur BudapestDunaföldvár est irréprochable. Mais si nous considérons, sur le secteur à l'aval de Budapest, la profondeur de $35 \mathrm{dm}$ en dessous du plus bas niveau de navigation (de régularisation) et si nous tenons compte des profondeur's moyennes actuelles sur les bas-fonds les plus hauts, la situation se présente sous un aspect bien plus défavorable.

Cependant, la réalisation d'un chenal de navigation plus profond que $25 \mathrm{dm}$ n'est, sur les autres secteurs du Danube aussi, qu'une tâche de l'avenir. II n'est quand même pas inutile d'en tenir compte lors des travaux en cours et de prévoir sur ce secteur, vu que ceux-ci contribuent à diminuer le danger d'embâcles, à augmenter la sécurité de la défense contre les crues, et à prolonger la saison de navigation, et qu'ils aboutissent à la diminution du besoin en force de traction. Pour cette raison, on a dressé des projets d'ouvrages de régularisation et construit ceux-ci de telle facon qu'ils n'entravent pas plus tard, mais aident plutôt la construction d'un chenal de navigation plus profond.

La situation changera plus tard lorsque la canalisation du Danube sera réalisée aussi sur ce secteur. En dressant le projet de la chute de Nagymaros, on a démontré que sur le secteur touché par la chute, le coût de l'exploitation de la navigation diminuera de $11 \%$,

Le secteur Szob-Mohács est un secteur de plaine à pente faible; sa canalisation et la mise en valeur de son énergie hydraulique recèlent donc beaucoup de difficultés. Ácluellement, c'est à l'extrémité amont du bras gauche fermé du Danube à l'île de Csepel que l'énergie hydraulique est tant soit peu ulilisée, en produisant quelque 6 millions de $\mathrm{kWh}$. Mais sur tout le secteur du Danube exposé dans la présente, la mise en valeur de l'énergie hydraulique du fleuve n'est qu'à l'état de décision de projet et de construction à échéance en partie proche, en partie lointaine.

Par comparaison avec les quelque dix-neuf chutes projetées sur le Haut Danube allemand et avec les treize chutes sur son secteur autrichien, ce n'est qu'avec bien moins de chutes que l'on peut envisager sur le Moyen Danube la mise en valeur de l'énergie hydraulique.

Après les chutes et les utilisations de l'énergie hydraulique projetées à Wolfsthal et à Bratislava sur le secteur de transition du Moyen Danube après la percée de Devin, c'est quatre chutes qu'il parait rationnel d'intercaler - en tenant compte des possibilités -. sur le secteur Szob-Mohács. Celles-ci fourniraient, en utilisant une chute totale de $32 \mathrm{~m}$ - chacune de 7-9 $\mathrm{m}$ au maximum annuellement quelque 2,6 milliards de $\mathrm{kWh}$. Parmi elles, c'est la chute projetée à Nagymaros, utilisant $9 \mathrm{~m}$ au maximum et fournissant annuellement en moyenne 810 millions de $\mathrm{kWh}$, qui est la plus proche de la réalisation. C'est ici que les conditions de construction sont les plus favorables, tandis que les trois autres chutes à Adony, Fajsz et Mohács (voir fig. 1) seront à construire dans des conditions beaucoup plus difficiles. Le grand avantage de l'aménagement hydraulique de Nagymaros est qu'il peut se construire avec accumulation de l'énergie par pompage, grâce aux possibilités très avantageuses qui existent dans les montagnes de la percée de Nagymaros.

Mais il faut encore mentionner la possibilité d'utiliser l'eau qui se présente sur le secteur SzobMohács, surtout au point de vue de l'approvisionnement en eau. Le cône de déjection s'étant formé à l'aval de la percée de Nagymaros se compose de bonnes couches aquifères, qui constituent une excellente source pour l'approvisionnement en eau des contrées riveraines. Le long du secteur, on peut rationnellement compter sur un prélèvement d'eau de $35-40 \mathrm{~m}^{3} / \mathrm{s}$ à obtenir avec «filtrage par les rives.». L'approvisionmement en eau potable et industrielle de Budapest se fait en majeure partie par les puits établis sur les rives s'échelonnant sur une distance de $25 \mathrm{~km}$ et seulement dans une pro- 


\section{IHRIG}

portion faible, en prélevant l'eau du Danube à la surface. Mais les chutes projetées dans l'avenir auront aussi une grande importance au point de vue de l'approvisionnement en eau de l'agriculture, puisqu'elles permettront l'irrigation de quel- que 170000 ha en fournissant l'eau par gravitation. Par ailleurs, le canal Danube-Tisza projeté, partant immédiatement à l'aval de Budapest, approvisionnerait en eau d'irrigation même la rivière Tisza et sa vallée.

\section{Zusammenfassung}

\section{Fragen der Regulierung und Nutzbarmachung der Donaustrecke Szob-Mohács Von D. lhrig *}

1. Beschreibung und Schilderung der Strecke. - Diese Donaustrecke ist der zwischen Ipolymündung $[F \mathrm{~km} 1708]$ und ungarisch-jugoslavischer Grenze liegende, gefällsarme Flachlandteil der das Karpathenbecken durchfliessenden sog. Mittleren Donau [Bild 1]. Sie beginnt bei der Ipolymündung mit einer zur Zeit schon vollständig reifen Durchbruchsstrecke [Bild 2]. Unterhalb des Durchbruchs trennt sich die Donau auf dem einstigen Schuttkegel in zwei Arme. Der Charakter des Stromes ist verzweigend, sich verbreitend. Bei Budapest fliesst er in durch Regulierungen zusammengeschnürtem Bett durch das Weichbild der Stadt bis zu deren südlichem Drittel [Bild 3]. Da spaltet sich die Donau wieder in zwei Arme.

Das Bild des Stromes verändert sich aber im unteren Drittel, bei Paks [Fkm 1533], bildet grosse Schleifen, nach deren Abschnürung die abgetrennten Betteile Inseln umfassen [Bild 4]. Die Bilder 5 und 6 zeigen je eine Ansicht der oberen und unteren Teile dieser Stromstrecke.

2. Die Regulierung der Strecke beeinflussende Gegebenheiten. - Am oberen zweidrittel Teil der Donaustrecke ist das Gefälle 8-9, im unteren Drittel 6-7 cm je Kilometer. Der Abfluss beträgt 500-10000, im Mittel $2120 \mathrm{~m}^{3} / \mathrm{s}$, Nebenflüsse gibt es auf der ganzen Strecke keine. Das Bettmaterial ist im oberen Zweidrittelteil schwer lösbarer Kies, im unteren Drittel dagegen leicht schürfbar und fein. Das Geschiebe ist oberhalb Budapest noch von Nussgrösse, bei Mohács nur noch millimetergross und dabei kaum auffindbar. Unterhalb Paks [Fkm 1523] befördert die Donau ihre Schwerstoffe schon grösstenteils als Schweb.

Nach der Verschiedenheit des Bettmaterials und der Geschiebeverhältnisse ist die Strecke in zwei Teile trennbar [S. Bild 1],

1. in der geschiebeführenden, stabil gebetteten Strecke oberhalb Paks und

2. der meandernden, schwebstofführenden Flachlandstrecke mit beweglichem Bett unterhalb Paks. Den oberen

Teil kennzeichnen lange gerade Strecken, das sich verbreitende, verzweigende Bett, den unteren Teil aber das

schlängede, gut eingebettete, zur Vertiefung neigende Flussbett.

3. Regulierung auf Mittel- und Niedrigwasserstand. - Das Hauptziel der Mittelwasser-Regulierungsarbeiten war in der Vergangenheit die Sicherung eines ungehemmten Eisabganges. Der Fluss war in seinem Naturzustand, wie ihn das Bild 4. darstellt, stark zur Bildung von Eisstössen geneigt, welche dann verheerende Hochwässer verursachten. Eins dieser war das Eishochwasser vom Jahre 1838, welches auch Budapest zum grossen Teil verheerte.

Mit den in der zweiten Hälte des vergangenen Jahrhunderts begonnenen Regulierungsarbeiten wurden die grossen Schleifen durchstochen und zuerst die Strecke bei Budapest und Umgebung reguliert, sodann schritt die Arbeit bis zur Jahrhundertswende bis Fajsz $[\mathrm{Fkm} 1508]$ vor. Der erste zusammenhängende Regulierungsplan der Mittleren Donau wurde 1893 angefertigt. Nach dem zweiten Weltkrieg wurde ein, den veränderten Verhältnissen und Uimständen entsprechender neuer Regulierungsplan verfasst.

Bei den neuen Entwürfen werden zur Bestimmung des Regulierungspiegels, d.h. des dem entsprechenden Schiffahrts-Niedriowassers gemäss des Vorschlags der Donaukommission jene Stände der 7 Hauptpegel der Strecke herangezogen, deren Dauer $94 \%$ beträgt. Die Regulierungsbreite ist bei den neueren Plänen $400 \mathrm{~m}$ [bei den Inflexionen 400 , beim Scheitel der Krümmungen $350 \mathrm{~m}$ ] und die Regulierungstiefe beträgt oberhalb Budapest $3,00 \mathrm{~m}$, bei und unterhalb Budapest $3,50 \mathrm{~m}$, welche Tiefe zur Zeit nicht nur die Ansprüche der Schiffahrt mit einem Ausblick auf die Zukunft befriedigt, sondern auch vom Gesichtspunkt des Eisabganges genügend Sicherheit bietet. Einen Teil der Regulierungen des oberen, stabil gebetteten Teils der Strecke veranschaulicht Bild 7.

4. Die Hochwasserregulierung. - Bild 8 charakterisiert die Eishochwässer der ungarischen Donaustrecke, vom Eis der Donau geben die Bilder 9 und 10 einen Begriff. Dem Schaubild gemäss übersteigt die Höhe der Eishochwässer der Donaustrecke unterhalb Budapest das höchste eisfreie Hochwasser bedeutend [mit 2-3'm]. Deshalb kann das gestaute Wasser bei Eisstossbildungen oft nur so unterhalb der zugelassenen Höhe abfliessen, dass es sich ausserhalb des verstopften Strombettes einen Weg sucht. So gelangt auf dieser Strecke die Regulierung des Hochwasserbettes zu sehr grosser Bedeutung. [Bild 7 weist auch eine solche Hochwasserbettkorrektion mit Verlegung des Schutzdammes auf].

5. Mit der Regulierung der Strecke zusammenhängende Wassernutzungstragen. - Die Hauptnutznehmer der Donau als Wasserlauf sind die Schiffahrt, die Kraftgewinnung und die Wasserversorgung.

Haupthindernisse der Schiffahrt sind das Eis und die Furten. Wegen der Eiserscheinung beträgt die durchschnittliche Schiffahrtszeit 321 Tage, die volle Ausnützung dieser Zeitspanne hindern aber die trotz Fortschreiten der Regulierungsarbeiten noch immer vorkommenden, weniger als $25 \mathrm{dm}$ tiefen Furten. Untiefen werden auf den Strecken oberhalb Budapest und unterhalb Dunaföldvár angetrofien, während die Strecke Budapest - Dunaföldvár von diesem Standpunkt tadellos ist.

Was die Kanalisierung [Abstaffelung] der Donau betriff, so sind Entwurf und Bauvorbereitung der ersten ungarischen Staustufe Nagymaros auf der Strecke Szob-Mohács schon vorgeschritten. Im Lauf der perspektiven Entwicklung sind noch fernere drei Staustufen: bei Adony, Fajsz und in der Nähe von Mohács geplant. Das Wasserkraftwerk Nagymaros kann mit dem Bau eines Pumpspei chers verbunden werden.

* Abteilungsleiter der Forschungsansalt für Wasserwirtschaft, Budapest. 